\title{
Ensino de matemática em cursos em ciências biológicas e da área da saúde: percepções de estudantes de doutorado
}

\author{
Mathematics teaching in biological sciences and \\ health area courses: doctoral students' perceptions
}

\author{
Janielle da Silva Melo da Cunha ${ }^{1}$ \\ Jéssica Maurino dos Santos ${ }^{2}$ \\ Tamaeh Monteiro Alfredo ${ }^{3}$ \\ Aldrin Cleyde da Cunha ${ }^{4}$
}

\begin{abstract}
RESUMO: Este artigo descreve as representações sociais de estudantes de doutorado da Universidade Federal da Grande Dourados, com relação ao ensino de matemática e estatística em cursos de graduação de Ciências Biológicas e da área da Saúde. Para coletar os dados realizamos entrevistas semiestruturadas, com dez estudantes de doutorado, no período de fevereiro a maio de 2018 , com aplicação de um roteiro com oito perguntas fechadas, considerando as teorias das Representações Sociais e Aprendizagem Inteligente como suporte teórico. A discussão do resultado foi organizado, com base no método do discurso do sujeito coletivo e ferramentas do QualiquantiSoft. Os participantes do estudo não conseguem relacionar os conteúdos matemáticos ensinados nas disciplinas de Matemática e Estatística em seus cursos de graduação, com suas práticas profissionais e relataram que tiveram dificuldades na aprendizagem. Além disso, os estudantes não percebem a aplicação dos conteúdo matemático à Ciência Biológica ou Ciência da Saúde e eles foram unânimes em afirmar que a matemática é um instrumento para resolução de cálculos que envolvem problemas biológicos. As representações sociais apresentadas no discurso do sujeito coletivo nos direcionam a refletir que no ensino superior, ainda prevalece o ensino da matemática instrumental e pouco desenvolvimento da compreensão e matemática relacional na sala de aula.
\end{abstract}

Palavras-chave: Educação Matemática; Ensino Superior; Matemática e Estatística.

ABSTRACT: This paper describes the social representations of doctoral students at the Federal University of Grande Dourados, for the teaching of Mathematics and Statistics in undergraduate courses in Biological Sciences and Health Area. To collect the data, we conducted semi-structured interviews, with ten doctoral students, from February to May 2018, with the application of a script with eight closed questions, considering the theories of Social Representations and Learning Intelligent as theoretical support. The discussion of the result was organized, based on the method of the collective subject discourse and tools of the Qualiquantisoft. The study participants cannot relate the mathematical contents taught in Mathematics and Statistics in their undergraduate courses, with their professional practices and reported that they had difficulties in learning. Besides, students do not perceive the application of mathemati-

\footnotetext{
${ }^{1}$ Doutora em Biotecnologia e Biodiversidade pela Universidade da Grande Dourados, professora assistente da Universidade Federal do Amapá, Curso de Licenciatura Intercultural Indígena, pesquisadora do Grupo de Estudos em Biotecnologia e Bioprospeç̧ão Aplicados ao Metabolismo e do Núcleo de Pesquisa História da Ciência e Ensino, E-mail: janiellecunha@hotmail.com

${ }^{2}$ Mestra em Biologia Geral/Bioprospecção pela Universidade da Grande Dourados, pesquisadora do Biotecnologia e Bioprospecção Aplicados ao Metabolismo, E-mail: jessy_maurino.s2@hotmail.com

${ }^{3}$ Doutoranda do Programa de Pós-Graduação em Ciências da Saúde pela Universidade da Grande Dourados, pesquisadora do Biotecnologia e Bioprospeç̧ão Aplicados ao Metabolismo, E-mail: tamaehmonteiro@hotmail.com

${ }^{4}$ Doutor em Educação Matemática pela Universidade Bandeirantes de São Paulo e professor adjunto da Universidade Federal de Grande Dourados, lotado na Faculdade Intercultural Indígena e pesquisador do Grupo Teia de Pesquisa em Educação Matemática, E-mail: aldrincunha@hotmail.com
} 
cal content to Biological Science or Health Science, and they were unanimous in stating that mathematics is an instrument for solving calculations involving biological problems. The social representations presented in the discourse of the collective subject direct us to reflect that in higher education, the teaching of instrumental mathematics still prevails, and little development of comprehension and mathematics relational in the classroom.

Keywords: Mathematics Education; Higher Education; Mathematics and Statistics.

\section{INTRODUÇÃO}

O conhecimento matemático insere-se em diferentes ciências, mas nem sempre é reconhecido sua aplicação para formular os modelos matemáticos.

Desde que nascemos construímos conhecimentos e aprendemos a retirar energia dos alimentos, o balanceamento energético que nosso corpo precisa para realizar desde pequenos movimentos e até mesmo para sobreviver. Mas quando classificamos estes saberes como parte do conhecimento científico, nos remetemos aos conteúdos ensinados nas escolas e universidades ou práticas de ensino, com abordagem disciplinar, interdisciplinar ou transdisciplinar.

Segundo Fourez (2001), a prática interdisciplinar utiliza as disciplinas para estudar uma situação em sua singularidade. No entanto, de que forma se podem trabalhar os conceitos da matemática nas diversas áreas da Biologia usando uma perspectiva interdisciplinar?

O enfoque interdisciplinar, de acordo com Lück (2007), "consiste num esforço de busca da visão global da realidade, como superação das impressões estáticas, e do hábito de pensar fragmentador e simplificador da realidade".

A orientação especializada que existe na pesquisa científica também reflete na formação para o exercício do magistério, podendo levar os professores em formação a considerar saberes de diferentes campos científicos como elementos dissociados e distanciados.

Neste contexto, investigações voltadas para o aprender e o compreender são discutidas no intuito de sistematizar relações que abrangem a matemática instrumental (saber fazer, mas sem necessariamente ter clareza a respeito do porquê e do que está envolvido naquilo que se está fazendo) e a matemática relacional (saber tanto o que fazer, quanto o porquê), esclarecer quais seus vínculos e diferenças, identificar e propor situações, bem como avaliar resultados em que contextos matemáticos são trabalhados (SKEMP, 1989).

O aprendizado por meio do hábito, associado à compreensão instrumental, segundo Skemp (1989) contribui para que o estudante se torne cada vez mais dependente de um professor que trabalha com regras, memorização e cálculos na matemática instrumental.

Já a aprendizagem inteligente desenvolve a confiança do estudante em suas próprias habilidades para enfrentar as dificuldades, quando se depara com situações matemáticas novas, o que envolve a compreensão e matemática relacional, desde que o pro- 
fessor o auxilie a ampliar a sua própria compreensão a respeito do assunto estudado (SKEMP, 1989).

O trabalho especializado dos professores que ensinam diferentes ciências no Brasil se fundamenta em currículos fragmentados e sem ligações entre as áreas de conhecimento. A fragmentação de saberes e o grande número de tópicos de ensino fez com que se mantivessem intactas e praticamente intransponíveis as fronteiras entre campos de saber excessivamente delimitados (CHAVES et al., 2008).

Este fato é bem referenciado por Kolling (2017), quando fala de questões mais significativas do que trabalhar com disciplinas, como os propósitos humanos, sociais e ambientais que contribuem não apenas com o conhecimento do indivíduo, mas com uma aprendizagem que será benéfico de forma coletiva.

Temos que considerar que os objetos de estudos da Educação Matemática são diversos, com base nas teorias produzidas nesta área de pesquisa ou outras. Neste campo de investigação, a Teoria das Representações Sociais (MOSCOVICl, 2001) ganha espaço e o estudo sobre percepções do ensino de matemática no ensino superior desenvolvem-se na pesquisa em educação matemática.

\section{MÉTODO E MATERIAL}

Para coletar os dados realizamos entrevistas semiestruturadas, com dez estudantes de doutorado da Universidade Federal da Grande Dourados - UFGD, no período de fevereiro a maio de 2018. Com base no aporte teórico da Teoria das Representações Sociais (MOSCOVICl, 2001), os dados foram analisados qualitativa e quantitativamente, com aplicação do método do discurso do sujeito coletivo - DSC (LEFEVRE, 2005).

Moscovici (2001) designa de Teoria das Representações Sociais (RS), o conjunto de ideias que estabelecem uma síntese teórica entre fenômenos que, em nível da realidade, estão profundamente ligados. Assim, as dimensões cognitivas, afetivas e sociais estão presentes na noção de representação social.

Com relação ao seu conceito, Gilly (2001) considera:

as representações como um grande sistema organizado de significação, úteis para a compreensão do que ocorre na sala de aula (presencial ou virtual), em relação às interações educativas, tanto do ponto de vista dos objetos de saber ensinados, quanto dos mecanismos psicossociais em ação no processo de aprendizagem (GILLY, 2001, p. 20).

O processo de elaboração das representações sociais nos direciona à atividade do sujeito social e seu pertencimento a grupo de indivíduos. Assim, nos estudos de representações sociais, o foco não se refere a um sujeito, "mas sim as respostas individuais enquanto manifestações de tendências do grupo que pertença ou de afiliação na qual os indivíduos participam (JODELET, 2001, p. 34).

A metodologia do discurso do sujeito coletivo - DSC é um conjunto harmônico de 
processos e procedimentos destinados, a partir de depoimentos colhidos em pesquisa sociais de opinião, reunindo, descritivamente, a opinião de uma dada coletividade como produto qualiquantitativo, isto é, como um painel de depoimentos discursivos (LEFEVRE, 2005).

As questões das entrevistas semiestruturadas estão descritas a seguir:

1. Qual a sua área de formação?

2. Durante a sua formação você teve alguma disciplina específica de matemática ou estatística?

3. Você teve dificuldade em compreender estas disciplinas?

4. Por quê?

5. Você considera que esta disciplina foi importante para sua formação?

6. Como você acha que deveria ser o ensino de matemática no seu curso?

7. Em sua atuação consegue aplicar conceitos matemáticos?

8. O que você acha correto afirmar: Os fenômenos biológicos são explicados por modelos matemáticos ou a matemática é utilizada como instrumento para a resolução de problemas e cálculos da Biologia.

Após a aplicação dos questionários, os discursos foram construídos a partir das ferramentas do Qualiquantisoft. Este software foi desenvolvido pela empresa Sales e Paschoal Informática, em parceria com a Universidade de São Paulo (USP), pelos professores Fernando Lefevre e Ana Maria Cavalcanti Lefevre e que são os criadores do método do discurso do sujeito coletivo - DSC (USP, 2007).

\section{DISCUSSÃO DOS RESULTADOS}

As áreas de formação dos entrevistados são apresentadas no Gráfico 1, o que caracteriza o perfil escolar dos participantes desta pesquisa, em se tratando de suas formações em nível de graduação.

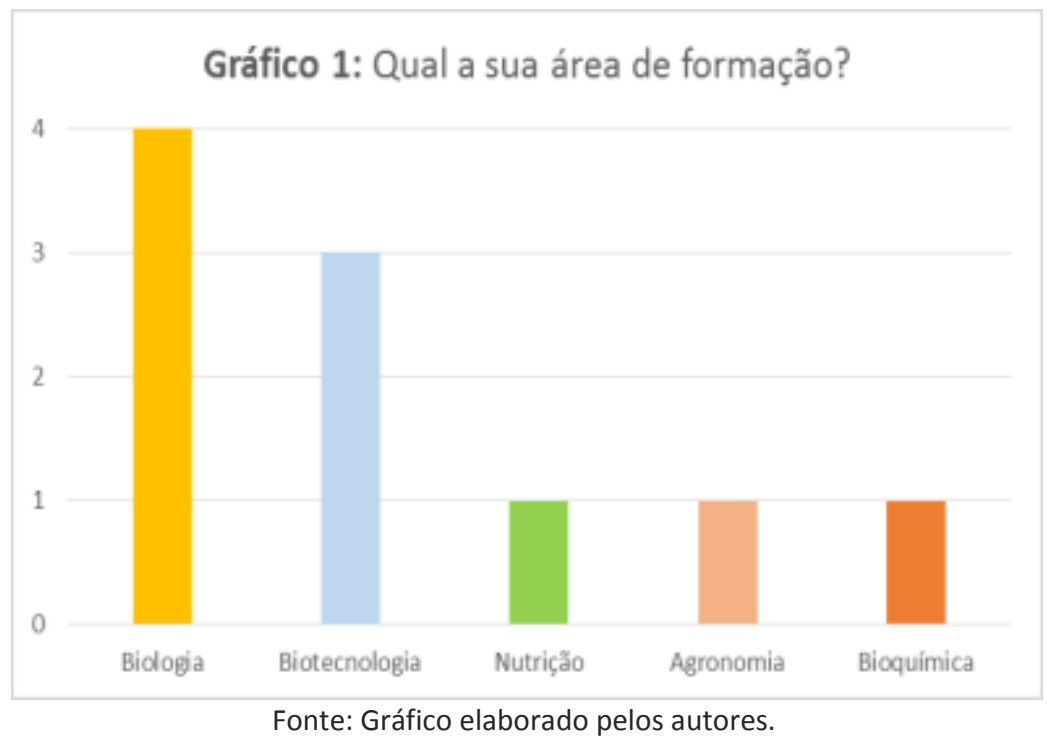


De acordo os dados coletados durante as entrevistas, todos os estudantes cursaram as disciplinas Matemática ou Estatística na sua formação acadêmica, o que é esclarecido no Gráfico 2. Além disso, os estudantes foram unânimes em responder que tiveram dificuldade em compreender o que foi ensinado nestas disciplinas nos cursos de pós-graduação stricto sensu, dado quantitativo que apresentamos no Gráfico 3.
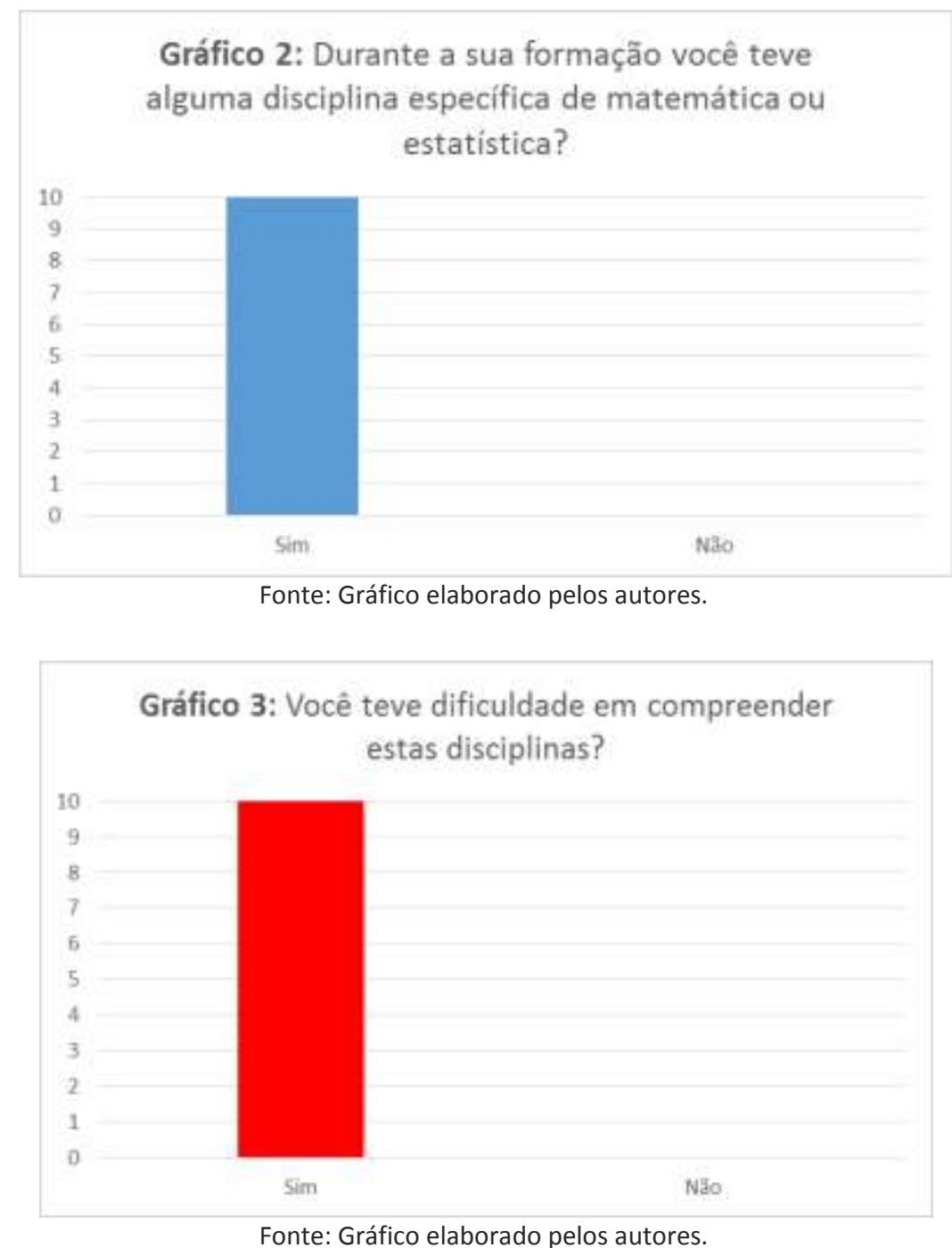

Os estudantes justificaram em seus discursos, que o professor que ministrou a disciplina de Matemática ou Estatística não tinha formação em Ciências Biológicas. Este é o fator que eles acreditam que leva o professor a não mostrar a aplicabilidade dos assuntos com sua futura profissão

Este aspecto da coleta dados é indicado no Gráfico 4, cujos dados quantitativos correspondem a $70 \%$ das respostas dos estudantes. Por outro lado, 30\% afirmaram que sua dificuldade deve-se aos muitos cálculos e deduções que se faz nestas disciplinas no ensino superior. 


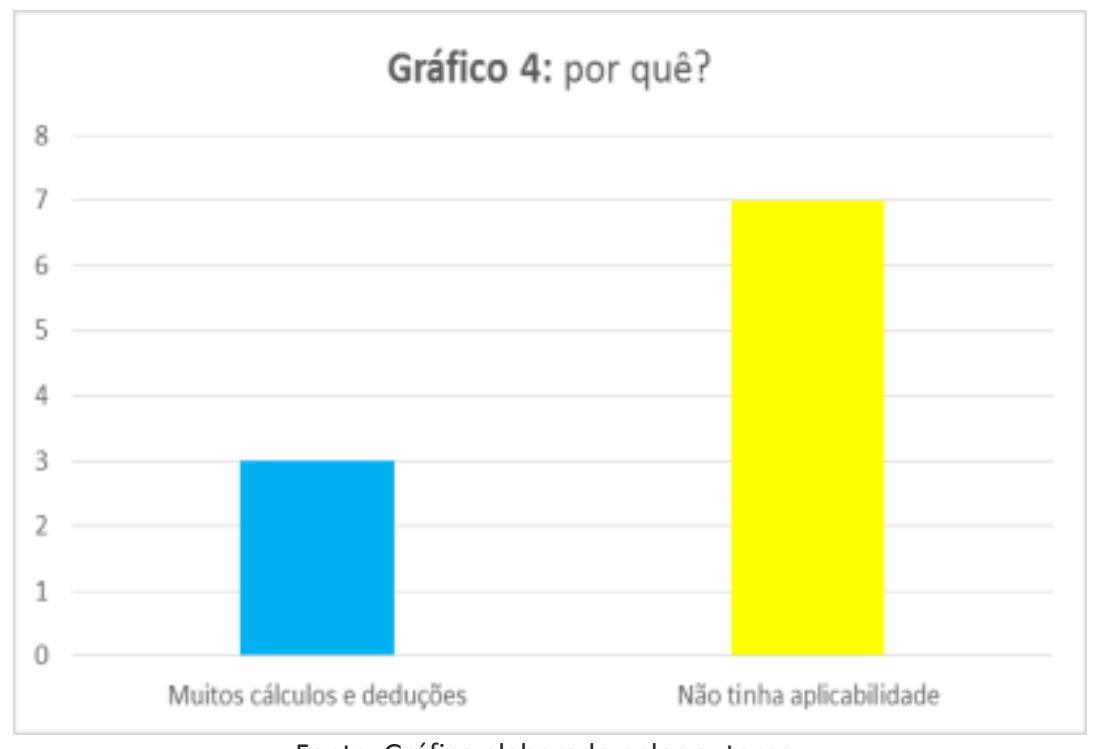

Fonte: Gráfico elaborado pelos autores.

Para essas respostas foram organizamos, os seguintes discursos do sujeito coletivo:

\section{Categoria A - Muitos cálculos e deduções.}

DSC: Além de ser uma disciplina de ciências exatas, foram realizados muitos cálculos e alguns estudantes tiveram dificuldades de entender os modelos estatísticos ou o professor não consegue explicar de maneira que todos os estudantes compreendessem o assunto.

\section{Categoria B - Não tinha aplicabilidade.}

DSC: A disciplina ministrada foi pouco aproveitada, visto que não era voltada à área de formação e, porque o professor da disciplina era estatístico e não conseguia mostrar a aplicabilidade.

Conforme Sacristán (2013), o currículo tem o sentido de constituir a carreira do estudante, consistindo em um plano de estudos ordenados relacionado aos conteúdos trabalhados. O que se percebe é que nos cursos das áreas biológicas e da saúde, as disciplinas de matemática e estatística são lecionadas por professores dos cursos de exatas. Isso nos leva a uma reflexão sobre até que ponto (e se ele existe), este professor tem conhecimento do perfil do estudante que ele está formando.

De acordo com o Gráfico 5, 60\% dos estudantes avaliam que a disciplina ministrada no seu curso de pós-graduação stricto sensu é importante para sua formação acadêmica. Por outro lado, $40 \%$ dos estudantes responderam que não, o que mostra que há divergências sobre o que os estudantes pensam acerca das disciplina de Matemática ou Estatística como parte do currículo no ensino superior. 


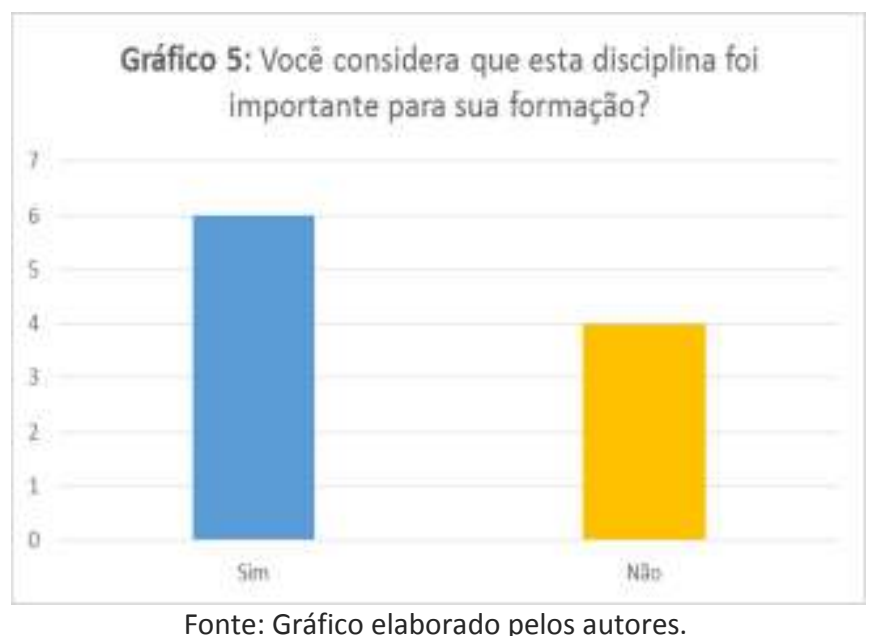

Ao serem questionados sobre como deveria ser o ensino de matemática nos cursos de Ciências Biológicas e da Saúde, $60 \%$ dos estudantes expressaram que o ensino dos assuntos relacionados à Matemática devem ter aplicações, com foco na área do curso stricto sensu, enquanto que $40 \%$ responderam que deve ser abordado as aplicações estatísticas, conforme exemplificado no Gráfico 6.

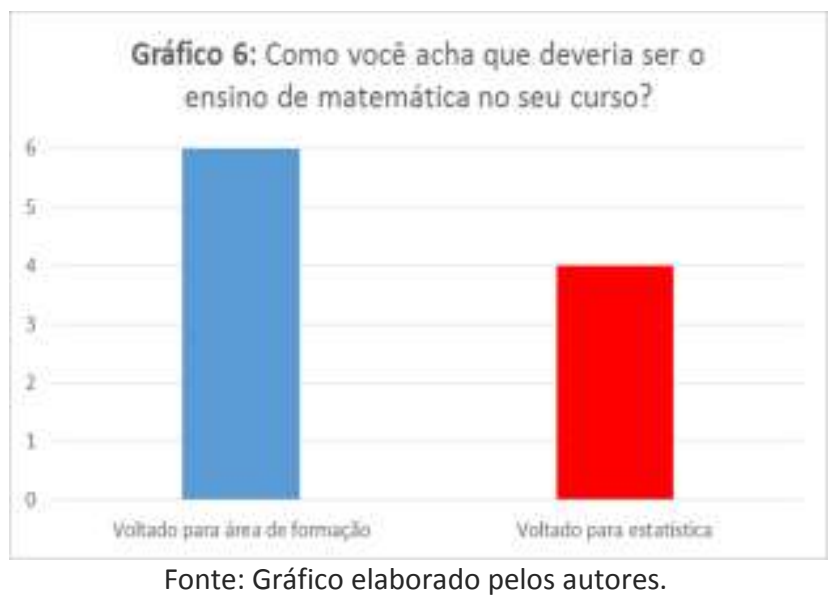

Os discursos dos sujeitos coletivos construídos para estas respostas foram:

\section{Categoria A - Voltado para área de formação.}

DSC: Mais específica, deveria ser aplicado a área do curso e apresentado aos estudantes dos cursos de pós-graduação, como uma ferramenta profissional e voltada para área de formação.

\section{Categoria B - Voltado para estatística.}

DSC: Voltada e direta para área da Estatística.

No que se refere ao campo da educação, Garcia (2008) define que:

[...] a interdisciplinaridade deve ser interpretada como uma "construção de pontes" entre conteúdos de diferentes disciplinas do currículo. Neste sentido o autor ainda 
indica que a interdisciplinaridade foi encarada na perspectiva epistemológica, buscando o diálogo integrativo de conceitos e na perspectiva relacionada ao desenvolvimento relacionado ao currículo da educação básica, na forma de estratégias para a integração entre disciplinas, aqui entendidas como as matérias do currículo escolar. Levando em conta a segunda perspectiva, torna-se importante salientar a exigência na forma de pensar e reestruturar o currículo, de forma que não passará mais a aceitar as especificidades do modelo tradicional (GARCIA, 2008, p. 5)

Assim, percebemos que a discussão no campo da educação, que já se estende a algumas décadas no Brasil, ainda precisa de práticas efetivas para consolidar um ensino que já não passa mais pela interdisciplinaridade e sim, pela transdisciplinaridade.

Etimologicamente, "trans" é o que está ao mesmo tempo entre as disciplinas, através das diferentes disciplinas e além de todas as disciplinas, remetendo também à ideia de transcendência. A transdisciplinaridade é uma teoria do conhecimento, é uma compreensão de processos, é um diálogo entre as diferentes áreas do saber e uma aventura do espírito. A transdisciplinaridade é uma nova atitude, é a assimilação de uma cultura, é uma arte, no sentido da capacidade de articular a multirreferencialidade e a multidimensionalidade do ser humano e do mundo (COLL, 2002 apud CUNHA, CUNHA, 2018, p. 26).

Segundo Cunha (2016), o essencial em uma formação transdisciplinar reside numa postura de reconhecimento que não há espaço e tempo culturais privilegiados que permitam julgar e hierarquizar, como mais correto ou mais certo ou mais verdadeiro, complexos de explicação e convivência com a realidade que nos cerca.

Quando questionados se os estudantes conseguiam aplicar os conceitos matemáticos em sua atuação profissional, mais uma vez, a resposta foi unânime, já que todos responderam que sim, conforme registado no Gráfico 7.

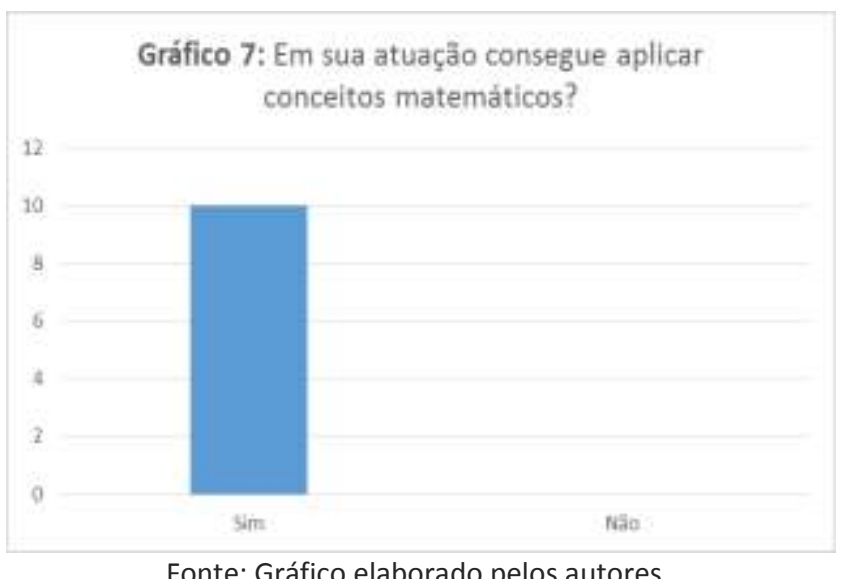

Fonte: Gráfico elaborado pelos autores.

Com relação à última questão da entrevista semiestruturada, de acordo com o Gráfico 8, todos os estudantes entendem que a matemática é aplicada apenas como um instrumento para a resolução de problemas e cálculos na área da Ciências Biológicas, o 
que revela que eles ainda não percebem que os fenômenos biológicos são explicados pelos modelos matemáticos.

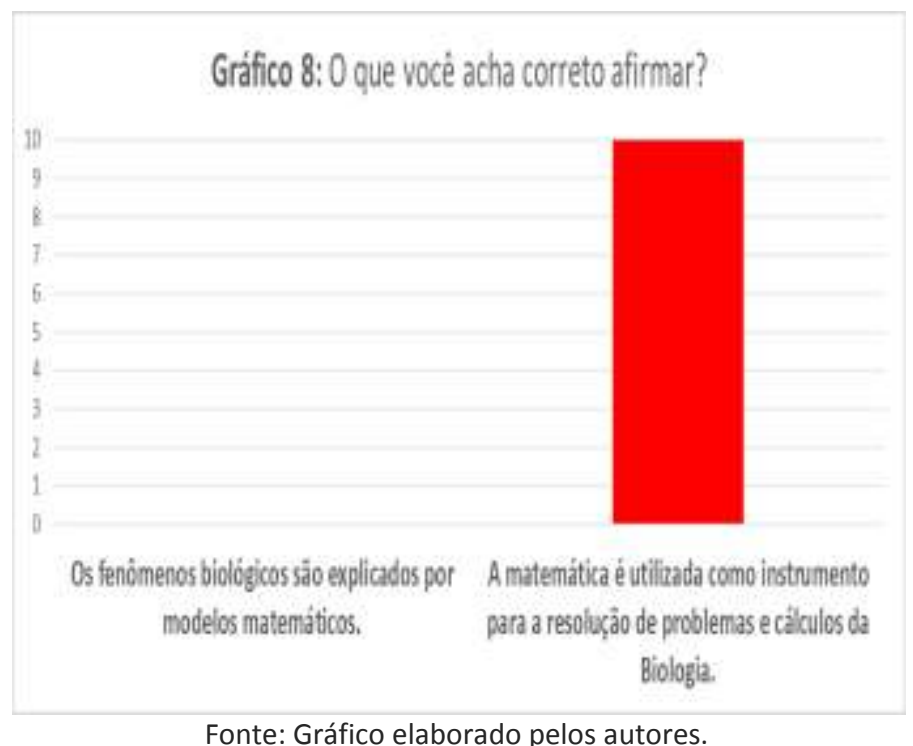

O diálogo entre as diferentes áreas do conhecimento no ensino superior, parece não ser explícito no processo ensino e aprendizagem, principalmente quando se refere a Ciência Biológica e Matemática, o que demanda por uma postura transdisciplinar por parte dos professores e estudantes dos cursos de pós-graduação.

A partir da prática transdisciplinar no ensino superior, baseada nos princípios básicos da ética da diversidade, "respeito, solidariedade e cooperação" (D'AMBROSIO, 2001), é possível aproximar os assuntos das Ciências Biológicas e Matemática para entender o que os professores ensinam e como eles ensinam na universidade, traz consequências à sociedade no futuro.

\section{CONSIDERAÇÕES FINAIS}

De acordo com o discurso do sujeito coletivo, os participantes do estudo não conseguem relacionar os conteúdos matemáticos ensinados em cursos de graduação, com suas práticas profissionais, além disso relataram que tiveram dificuldade na aprendizagem de matemática.

O grupo pesquisado considera importante a disciplina de Matemática à qualificação profissional, mas também destacaram a falta de aplicabilidade do conteúdo matemático à Ciência Biológica, assim como na área da Saúde.

Há de se considerar que o conhecimento matemático insere-se em diferentes áreas de pesquisa, no entanto, nem sempre é compreendido como parte da ciência que apoia a formulação de modelos matemáticos. Este aspecto evidenciou-se quando os estudantes durante as entrevistas, interpretam apenas a matemática como um instrumento para resolver problemas na área das Ciências Biológicas, conforme ilustrado no último 
gráfico da discussão dos resultados.

É necessário desenvolver interesses comuns no campo educacional e não só ensinar Estatística ou Matemática. Mas relacioná-las com temáticas de outras ciências para compreender que cada área do conhecimento tem seu papel na formação acadêmica e que áreas distintas conduzem o estudante a entender um objeto de estudo de forma abrangente.

As representações sociais destacadas no discurso do sujeito coletivo, nesta pesquisa em educação matemática, nos direcionam a refletir que no ensino superior ainda prevalece o ensino da matemática instrumental.

\section{REFERÊNCIAS}

CUNHA, A. C. Contribuição da etnomatemática para a manutenção e dinamização da cultura Guarani e Kaiowá na formação inicial de professores indígenas. (Tese de Doutorado). Universidade Anhanguera de São Paulo. São Paulo, 2016.

CUNHA, J. S. M; CUNHA, A. C. Etnomatemática no contexto da formação de professores indígenas na educação superior. Science and Knowledge in Focus, Macapá, v. 1, n.1, p. 19-38, 2018.

D’AMBROSIO, U. Transdisciplinaridade. São Paulo: Palas Athena, 2001.

FOUREZ, G. Fondements Epistémologiques pour l'interdisciplinante. In: LENOIR, Y.: REY, B.; FAZENDA, I. (Ed.) Les fondements de l'interdisciplinarité dans la formatoion à I'enseignement. Sherbrooke: Editions du CRP, 2001. p. 81-83.

GARCIA, J. A interdisciplinaridade segundo os PCNs. Revista de Educação Pública, Cuiabá, v. 17, n. 35, p. 363-365, Set-Dez. 2008.

GILLY, M. As representações sociais no campo da educação. In: JODELET, D. (Org.) As representações sociais. Trad. Lilian Ulup. Rio de Janeiro: EdUERJ, 2001.

JODELET, D. (Org.). As representações sociais. Trad. de Lilian Ulup. Rio de Janeiro: Ed. UERJ, 2001.

KOLLING, D. Como a transdisciplinaridade se manifesta no ensino de ciências e matemática, na licenciatura em pedagogia EAD. (Dissertação de Mestrado). Pontifícia Universidade Católica do Rio Grande do Sul. Porto Alegre, 2017.

LEFEVRE, F.; LEFEVRE, A. M. C. Depoimento e Discurso: uma proposta de análise em pesquisa social. Brasília: Liber Livro, 2005. (Série Pesquisa, 12).

LÜCK, H. Pedagogia Interdisciplinar: Fundamentos teóricos-metodológicos. Petrópolis: Vozes, 2007.

MOSCOVICI, S. Das representações coletivas às representações sociais: elementos para uma história. In: JODELET, D. (Org.). As representações sociais. Trad. de Lilian Ulup. Rio de Janeiro: Ed. UERJ, 2001. 
SACRISTÁN, J. G. O que quer um currículo? In: SACRISTÁN, José G (Org.) Saberes e Incertezas sobre o currículo. Porto Alegre: Penso, 2013.

SILVA JÚNIOR, G. B. Biologia e Matemática: Diálogos Possíveis no Ensino Médio. (Dissertação de Mestrado). Pontifícia Universidade Católica de Minas Gerais. Belo Horizonte, 2008.

SKEMP, R. R. Mathematics in the primary school. Londres: Routledge, 1989.

UNIVERSIDADE DE SÃO PAULO. Software ajuda a fazer pesquisa. Sala de Imprensa, São Paulo, 07 de março de 2007. Disponível em: http://www.usp.br/imprensa/?p=925, Acesso: 06/03/2018.

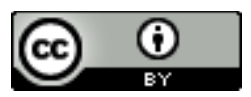
tive Commons Attribution License, which permits unrestricted use, distribution, and reproduction in any medium, provided the original work is properly cited.

Article received on March 06, 2018.

Accepted on October 23, 2018. 